\title{
The role of fear and expectancies in capture of covert attention by spiders
}

\author{
Christel Devue $^{1,2}$, Artem V. Belopolsky ${ }^{1}$ and Jan Theeuwes ${ }^{1}$ \\ ${ }^{1}$ Cognitive Psychology, Vrije Universiteit Amsterdam, the Netherlands \\ ${ }^{2}$ Centre de Neurosciences Cognitives et Comportementales, Université de Liège, Belgium
}

\section{In press in Emotion}

Correspondence to:

Christel Devue

Department of Cognitive Sciences

Université de Liège

Boulevard du Rectorat, 5 (Bât. B32)

B-4000 Liège

Belgium

Email: cdevue@ulg.ac.be

Tel.: +32[0]4 3662021

Fax: $+32[0] 43662859$ 


\begin{abstract}
Fear-related stimuli are often prioritized during visual selection but it remains unclear whether capture by salient objects is more likely to occur when individuals fear those objects. In this study, participants with high and low fear of spiders searched for a circle while on some trials a completely irrelevant fear-related (spider) or neutral distractor (butterfly/leaf) was presented simultaneously in the display. Our results show that when you fear spiders and you are not sure whether a spider is going to be present then any salient distractor (i.e., a butterfly) grabs your attention, suggesting that mere expectation of a spider triggered compulsory monitoring of all irrelevant stimuli. However, neutral stimuli did not grab attention when high spider fearful people knew that a spider could not be present during a block of trials, treating the neutral stimuli just as the low spider fearful people do. Our results show that people that fear spiders inspect potential spider-containing locations in a compulsory fashion even though directing attention to this location is completely irrelevant for the task. Reduction of capture can only be accomplished when people that fear spiders do not expect a spider to be present.
\end{abstract}

\title{
Keywords
}

Anxiety, Attentional selection, Bottom-up process, Covert attention, Spider phobia, Visual search 


\section{INTRODUCTION}

Imagine that while sitting in your office and reading this article a spider crawls up to you. Do you think that this spider would automatically grab your attention? And would its presence be more disruptive if you fear spiders? Research on visual attention has shown that salient stimuli can capture attention irrespective of our goals and intentions in a bottom-up, automatic fashion (Theeuwes, 1992, 2004; Yantis \& Jonides, 1984). Similarly, it has been suggested that potentially threatening stimuli, such as spiders and snakes, can also capture attention. The detection of fear-related stimuli is assumed to activate an evolutionary-driven inborn defense system, which prepares the individual to take action and to deal with the situation. Such automatic orienting response is assumed to reduce the presumed risk of being harmed by the fear-related stimulus (Mineka \& Öhman, 2002; Öhman, 1996; Öhman, Flykt, \& Esteves, 2001; Williams, Watts, MacLeod, \& Mathews, 1997). However, even though intuitively plausible, there is not much, if any, empirical evidence that unequivocally shows that fear-related objects capture our attention.

Previous research has shown an attentional bias towards fear-related stimuli such as spider-related pictures and words (e.g., Lipp \& Derakshan, 2005; Mogg \& Bradley, 2006; Rinck, Reinecke, Ellwart, Heuer, \& Becker, 2005; Vrijsen, Fleurkens, Nieuwboer, \& Rinck, 2009). For example, one is able to quickly find fear-related stimuli in a scene or among distractors (Pflugshaupt, Mosimann, von Wartburg, Schmitt, Nyffeler, \& Müri, 2005; Rinck \& Becker, 2006; Soares, Esteves, \& Flykt, 2009a; but see Lipp, Derakshan, Waters, \& Logies, 2004) and is distracted by a spider when searching for a neutral target (Lipp \& Waters, 2007; Miltner, Krieschel, Hecht, Tripp, \& Weiss, 2004; Rinck et al., 2005). Also, discriminating a fear-related object from background is often quicker than discriminating a neutral target (Öhman et al., 2001; Rinck et al., 2005; Soares, Esteves, Lundqvist, \& Öhman, 2009b; but see Miltner et al., 2004). Typically, these findings have been interpreted as evidence that fearrelated stimuli capture attention in an automatic bottom-up way (e.g., Lipp \& Waters, 2007; Öhman et al., 2001; Pflugshaupt et al., 2005; Rinck \& Becker, 2006; Waters \& Lipp, 2008; Williams et al., 1997).

Even though it is clear that processing fear-related stimuli is different from processing neutral stimuli, it has not yet been established whether fear-related stimuli capture attention when they are completely irrelevant for the task. In addition, it is unclear whether a heightened capture effect will be specific to the feared objects or extend more generally to any 
salient object. In most other studies conducted, fear-related stimuli were typically in some way task relevant. In the present study, the stimulus feature in question was never relevant for the task, so that there is no incentive for the observer to attend to it deliberately (Theeuwes, 2010; Yantis \& Egeth, 1999).

Furthermore, not all studies have found a processing advantage for spiders (Lipp, 2006; Miltner et al., 2004; Rinck et al., 2005). For example, a recent eye-movement study by Gerdes, Alpers and Pauli (2008) showed that an irrelevant onset of a spider does not capture the eyes more than an onset of a flower or a mushroom. Other studies have shown that spider targets were not found faster than neutral targets when they all appeared among neutral distractors but that the search for a neutral target was slower when it appeared among spider distractors (Lipp, 2006; Miltner et al., 2004; Rinck et al., 2005). A possible reason for these discrepancies in results is that it is not always easy to control for low level differences between fear-related and neutral stimuli. More specifically low-level feature differences between stimuli are inevitable, especially when using natural images. For example, a threatening object may have specific colours or appear on different backgrounds than neutral stimuli (see Cave and Batty, 2006 for a similar argument). Moreover, threatening objects like spiders are often compared to flowers, fruits or mushrooms which are all inanimate objects (see e.g. Gerdes et al., 2008; but see Rinck et al., 2005; Soares et al., 2009a). In line with this idea, some studies have found a search advantage for animals independently of their fearrelevance (Lipp et al., 2004; Lipp, 2006). Therefore, it is not always clear whether the observed attentional biases are due to fear-relevance per se or to the stimulus material that was used.

A first way to circumvent this problem is to examine people with a high-fear of well specified stimulus sets (see e.g. Lipp et al., 2004; Miltner et al., 2004; Rinck et al., 2005; Soares et al., 2009a, 2009b). Animal-specific phobias and especially arachnophobia are highly prevalent in the general population (Szymanski \& O’Donohue, 1995). Comparing people with and without fear of spiders thus offers a way to investigate the influence of fear on attentional selection, while controlling for all kinds of differences between stimuli. A second way is to also reduce as much as possible low (e.g. colour) and high-level (e.g. category) differences existing between fear-related and neutral stimuli, for instance by using schematic stimuli of different kinds of arthropods (e.g. spiders and butterflies). Silhouettes of spiders should produce a sufficient fear in people with high-fear because individuals with an extreme fear of 
spiders are often so scared of spiders that even verbally mentioning them or seeing schematic representations of them may already be highly distressful (Lang \& McTeague, 2009; Gerdes, Uhl, \& Alpers, 2009).

In the present study, we addressed the abovementioned concerns and employed the well-known additional singleton task of Theeuwes $(1991,1992)$ to investigate capture of covert attention by fear-related objects. In this task which has been extensively used to investigate attentional capture (see for a review Theeuwes, 2010), participants have to consistently search for a target circle among multiple diamond shapes and respond to the line segment inside the target circle. On some trials, an irrelevant object (usually a salient color singleton) is simultaneously presented and the increase in the time to find the target shape is considered to represent the extent of attentional capture by the irrelevant object (see Theeuwes, 2010). In the present study, instead of a color singleton, an irrelevant schematic fear-related (a spider) or neutral (a butterfly in Experiment 1, a butterfly or a leaf in Experiment 2) object was used. We measured the extent to which these objects that were completely irrelevant to the search task increased the time to find the target. Furthermore, to isolate the impact of fear on capture, we compared people with or without fear of spiders. Using participants with fear of spiders is not only important from a clinical point of view, but it also can establish that a difference in capture between these groups is specifically related to the experience of fear. We predicted that if fear-related stimuli can indeed capture covert attention and if the experience of fear drives this effect (see Lipp, 2006; Lipp \& Waters, 2007; Soares et al., 2009a) then the irrelevant presence of a spider should increase reaction times more than a butterfly, and should be more disruptive in the high-fear than in the low-fear group.

\section{EXPERIMENT 1}

\section{Method}

\section{Participants.}

We screened 367 students (319 females; mean age $=20$ years, $S D=4$ years) of bachelor in psychology at the University of Liège. They filled in the French version of the Fear of Spiders Questionnaire (FSQ, Delroisse \& Philippot, 2007) that comprises 18 questions assessing fear 
of spiders by means of 7-points Likert scales. A $19^{\text {th }}$ question assesses the impact of fear of spiders on daily life (i.e. "Do you consider that fear of spiders interferes with your daily life?"). In addition, students also rated their fear and disgust of a set of various animals including spiders and butterflies by means of 5-points Likert scales (1: "no fear/disgust", 5: “extreme fear/disgust"). Among the 367 respondents, 214 agreed to provide their details to be contacted for a later experiment.

For the high-fear of spiders group, we selected 24 participants (all females, mean age $=20.8$ years, $S D=1.8$ ) whose scores to the FSQ were within the $75^{\text {th }}$ percentile of the whole sample $(\geq 64)$ and who reported a fear and disgust of spiders above 3 and a fear and disgust of butterflies below 3. For the low-fear group, we selected 24 participants (20 females, mean age $=21.2$ years, $S D=3.7$ ) whose scores to the FSQ were within the $25^{\text {th }}$ percentile of the whole sample $(\leq 22.25)$ and who reported a fear and disgust of both spiders and butterflies below 3 . All participants had normal or corrected-to-normal vision and gave their informed consent before taking part in the experiment.

\section{Stimuli.}

The stimuli were green circles containing a vertical $\left(0.5^{\circ}\right)$ or horizontal line $\left(0.5^{\circ}\right)$, green diamonds containing one tilted line and black schematic animals (fitted within a $2^{\circ}$ square). There were 4 different spiders and 4 different butterflies composed on average of the same number of pixels (see Figure 1A). "No distractor" displays included 6 diamonds and 1 circle. "Distractor" displays included 1 circle, 5 diamonds and 1 schematic animal. These items were positioned on an imaginary circle at $3.4^{\circ}$ of eccentricity from the central fixation point $\left(0.3^{\circ}\right)$ and presented against a light gray background.

\section{Procedure.}

Participants were tested individually in a dimly lit room on a PC. They were seated $56 \mathrm{~cm}$ away from a 17-inch monitor as controlled by means of a chin-rest. They performed two different tasks in a counterbalanced order.

In the additional singleton task, participants searched from fixation for the unique circle and responded to the orientation of the line inside of it. There were 320 trials presented in a random order. On half of the trials, no distractor was present and on the other half of the trials an animal distractor (spider or butterfly) was present (20 times per exemplar). The position of the different types of items and the orientation of the different lines varied at random. Each 
trial began with a fixation dot $\left(0.3^{\circ}\right)$ presented for $1000 \mathrm{~ms}$, followed by a larger fixation dot $\left(0.7^{\circ}\right)$ presented for $600 \mathrm{~ms}$ to announce the upcoming display. The display was then presented until the participant responded by pressing one of the two buttons (" $\mathrm{S}$ " = vertical; "L" = horizontal) or up to $4000 \mathrm{~ms}$. A $500 \mathrm{~ms}$ blank during which an auditory feedback was played in case of incorrect or no response ended the trial (see Figure 1B). Short breaks were given every 40 trials. Before the experimental trials, participants received 80 training trials without a distractor.

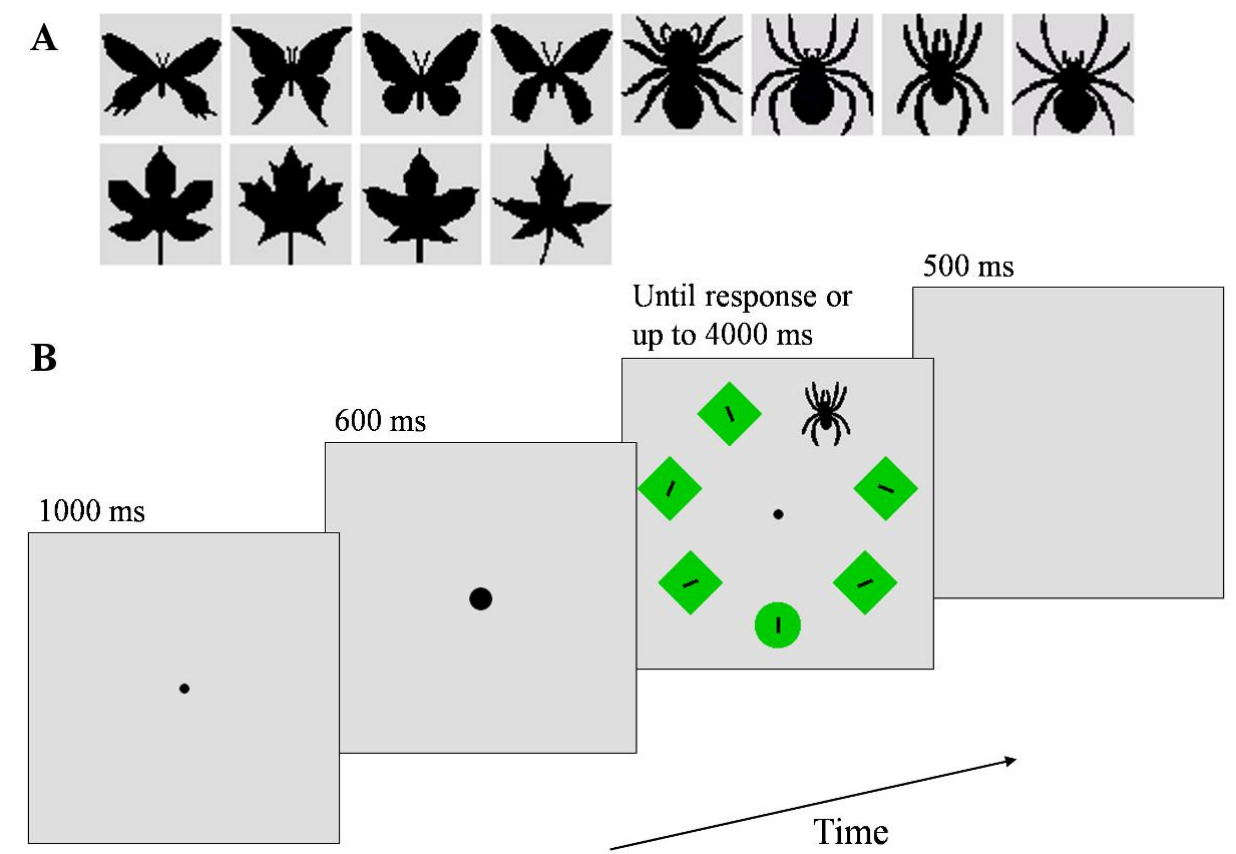

Figure 1. (A) Schematic stimuli used in Experiment 1 (butterflies and spiders) and in Experiment 2 (butterflies, spiders and leaves); (B) Illustration of the time course of a trial in the two tasks: In the additional singleton task (Exp. 1 and 2), participants were instructed to judge the orientation of the line inside the circle; in the perceptual discrimination task (Exp. 1), they were instructed to discriminate whether the animal was a spider or a butterfly.

As a second task we used a perceptual discrimination task to determine whether participants were able to discriminate between a spider and a butterfly from the central fixation point and to rule out explanations of possible differences between groups in the main task in terms of a general difference of sensitivity. This is important because if we want to make the claim that it is the content of the picture (spider versus butterfly) that drives attention then we should ensure that participants of both groups can indeed discern quickly and accurately the content of the picture. So even if high-fear participants might recognize spiders better than low-fear participants (i.e. threat superiority effect, see e.g. Fox et al., 2007), butterflies should be recognized as easily by both groups since they are neutral to both. The procedure was the 
same as in the main task except that participants were instructed to determine from fixation whether either a spider or a butterfly was presented by pressing one of two buttons ("B" or "N") whose order was counterbalanced across participants. There were in total 160 trials (20 trials per animal exemplar). Participants received a 24-trials training session before the experimental trials.

After the completion of the two tasks, participants filled in the Spider Belief Questionnaire (SBQ; Arntz, Lavy, van den Berg, \& van Rijsoort, 1993) to have an additional measure of fear of spiders. This questionnaire consists of 42 statements assessing, on 4-points scales (from "never" to "always"), the frequency of thoughts about spiders occurring in the presence of a spider and the strength of the belief in this thought (from 0 to 100\%). Moreover, 36 statements assess the frequency of thoughts about one's own reaction when facing a spider and the strength of the belief in those thoughts. In addition, participants filled in the French version of the State-Trait Anxiety Inventory (Y-form, Bruchon-Schweitzer \& Paulhan, 1993) and of the Beck Depression Inventory-13 items (Beck et al., 1974) in order to assess, respectively, their level of state and trait anxiety and of depression.

\section{Results}

\section{Questionnaires.}

Results presented on Table 1B confirm that high-fear participants scored higher than low-fear participants on all aspects of spider anxiety (i.e. FSQ, fear and disgust of spiders, and 4 measures of the SBQ). Delroisse and Philippot (2007) reported mean scores of 42.48 (i.e. 2.36 by 18) to the FSQ in an all-coming population. Even though they were not formally assessed for spider phobia, the mean score of our high-fear participants (i.e. 89.4) was in the range of the clinical threshold (i.e. 2 standard deviations above the mean, that is 81 ) as defined by the authors for women of this age group. Despite the fact that we only selected participants who reported no or only a slight fear/disgust of butterflies, high-fear participants scored higher on their level of fear of butterflies than low-fear participants. The level of fear of spiders of highfear participants was nonetheless much higher than their level of fear of butterflies, $t(23)=24.35 ; p<0.001$, and the level of fear of butterfly was actually lower than 2 ("slight"). Table 1A shows that participants of both groups did not differ significantly regarding their level of depression but that they tended to differ on their levels of State and Trait anxiety. 
Table 1. Questionnaire scores (Range of possible scores, Means - in bold, standard deviations - in italics, $t$-tests) in high- and low- fear of spiders groups in Experiment 1 and Experiment 2.

A Experiment 1

\begin{tabular}{llcccccc}
\hline & Range & High-fear & \multicolumn{3}{c}{ Low-fear } & $\boldsymbol{t}$ value & $\boldsymbol{p}$ \\
\hline STAI-S & $20-80$ & $\mathbf{3 5 . 9}$ & 10.8 & $\mathbf{3 0 . 5}$ & 8.3 & 1.95 & $=0.057$ \\
STAI-T & $20-80$ & $\mathbf{4 6}$ & 8.6 & $\mathbf{4 1 . 5}$ & 8 & 1.86 & $=0.07$ \\
BDI-13 & $0-39$ & $\mathbf{4 . 5}$ & 4 & $\mathbf{3 . 1}$ & 3.2 & 1.32 & $=0.19$ \\
\hline
\end{tabular}

Experiment 2

\begin{tabular}{llcccccc}
\hline & Range & High-fear & \multicolumn{3}{c}{ Low-fear } & $\boldsymbol{t}$ value & $\boldsymbol{p}$ \\
\hline STAI-S & $20-80$ & $\mathbf{4 2 . 5}$ & 12.4 & $\mathbf{3 0 . 4}$ & 8.3 & 2.8 & $<0.02^{*}$ \\
STAI-T & $20-80$ & $\mathbf{5 0 . 1}$ & 11.5 & $\mathbf{4 0 . 7}$ & 9 & 2.2 & $<0.05^{*}$ \\
BDI -13 & $0-39$ & $\mathbf{7 . 2}$ & 4.5 & $\mathbf{4 . 2}$ & 3.4 & 1.8 & $=0.08$ \\
\hline
\end{tabular}

B Experiment 1

\begin{tabular}{llccccccc}
\hline & Range & High-fear & & Low-fear & \multicolumn{1}{c}{ value } & \multicolumn{1}{c}{$\boldsymbol{p}$} \\
\hline FSQ 18-items & $18-126$ & $\mathbf{8 9 . 4}$ & 13.3 & $\mathbf{2 0 . 1}$ & 1.5 & 25.36 & $<0.001$ & $*$ \\
FSQ-DI & $1-7$ & $\mathbf{3 . 3}$ & 1.5 & $\mathbf{1}$ & 0 & - & - & \\
Fear of spiders & $1-5$ & $\mathbf{4 . 5}$ & 0.5 & $\mathbf{1 . 3}$ & 0.4 & 23.91 & $<0.001$ & $*$ \\
Disgust of spiders & $1-5$ & $\mathbf{4 . 5}$ & 0.5 & $\mathbf{1 . 4}$ & 0.5 & 21.53 & $<0.001$ & $*$ \\
Fear of butterflies & $1-5$ & $\mathbf{1 . 4}$ & 0.5 & $\mathbf{1}$ & 0.2 & 3.05 & $<0.005$ & $*$ \\
Disgust of butterflies & $1-5$ & $\mathbf{1 . 1}$ & 0.3 & $\mathbf{1}$ & 0 & - & - & \\
& & & & & & & & \\
SBQ - SpiderF & $1-4$ & $\mathbf{2 . 2}$ & 0.3 & $\mathbf{1 . 5}$ & 0.3 & 8.8 & $<0.001$ & $*$ \\
SBQ - SpiderI & $0-100(\%)$ & $\mathbf{3 5 . 1}$ & 11.6 & $\mathbf{1 3 . 5}$ & 10.7 & 6.6 & $<0.001$ & $*$ \\
SBQ - SelfF & $1-4$ & $\mathbf{2}$ & 0.5 & $\mathbf{1 . 2}$ & 0.2 & 7.4 & $<0.001$ & $*$ \\
SBQ - SelfI & $0-100(\%)$ & $\mathbf{3 3 . 4}$ & 19.3 & $\mathbf{4 . 3}$ & 5.8 & 6.9 & $<0.001$ & $*$ \\
\hline
\end{tabular}

Experiment 2

\begin{tabular}{llccccccc}
\hline & Range & High-fear & & Low-fear & \multicolumn{1}{c}{ value } & $\boldsymbol{p}$ & \\
\hline FSQ 18-items & $18-126$ & $\mathbf{9 8 . 5}$ & 16.5 & $\mathbf{2 0 . 7}$ & 2.6 & 16.1 & $<0.001$ & $*$ \\
FSQ-DI & $1-7$ & $\mathbf{3 . 9}$ & 2.3 & $\mathbf{1}$ & 0 & - & - & \\
Fear of spiders & $1-5$ & $\mathbf{4 . 8}$ & 0.4 & $\mathbf{1 . 3}$ & 0.5 & 19.3 & $<0.001$ & $*$ \\
Disgust of spiders & $1-5$ & $\mathbf{5}$ & 0 & $\mathbf{1 . 3}$ & 0.5 & 25.8 & $<0.001$ & $*$ \\
Fear of butterflies & $1-5$ & $\mathbf{1 . 1}$ & 0.3 & $\mathbf{1}$ & 0 & - & - & \\
Disgust of butterflies & $1-5$ & $\mathbf{1 . 1}$ & 0.3 & $\mathbf{1}$ & 0 & - & - & \\
& & & & & & & & \\
SBQ - SpiderF & $1-4$ & $\mathbf{2 . 6}$ & 0.4 & $\mathbf{1 . 3}$ & 0.2 & 9.7 & $<0.001$ & $*$ \\
SBQ - SpiderI & $0-100(\%)$ & $\mathbf{4 8 . 9}$ & 16.9 & $\mathbf{1 1 . 9}$ & 8.3 & 6.8 & $<0.001$ & $*$ \\
SBQ - SelfF & $1-4$ & $\mathbf{2 . 5}$ & 0.6 & $\mathbf{1 . 2}$ & 0.2 & 8 & $<0.001$ & $*$ \\
SBQ - SelfI & $0-100(\%)$ & $\mathbf{5 0 . 3}$ & 20.5 & $\mathbf{5}$ & 8.4 & 7.1 & $<0.001$ & $*$ \\
\hline
\end{tabular}

Note. (A) STAI-S = State-Trait Anxiety Inventory - State; STAI-T = State-Trait Anxiety Inventory - Trait; BDI-13 = Beck Depression Inventory. (B) FSQ 18-items = Fear of Spiders Questionnaire; FSQ-DI $=19^{\text {th }}$ question about the daily life impact of fear of spiders; SBQ $=$ Spider Beliefs Questionnaire, SpiderF = frequency of spiders-related beliefs; SpiderI = intensity of spiders-related beliefs; SelfF = frequency of self-related beliefs; SelfI = intensity of self-related beliefs. 


\section{Additional singleton task.}

In both groups, one participant was removed from the sample because they had a mean reaction time (RT) above $3.5 S D$ or error rates above $4 S D$ from the whole sample, respectively. We first conducted a two-way mixed effects analysis of variance (ANOVA) with Group (high-fear, low-fear) as between-subjects and Distractor presence (present, absent) as within-subjects factors on mean correct RTs to the additional singleton task (see Figure 2A). There was no main effect of Group, $F<1$, so overall RTs were not affected by the fear of spiders. As expected, there was a capture effect: the presence of an irrelevant distractor slowed RTs, as reflected by a main effect of the Distractor presence, $F(1,44)=80.35 ; p<0.001$. The interaction between Group and Distractor presence was significant, $F(1,44)=5.44$; $p<0.05$, suggesting that capture was larger for the high-fear than for the low-fear group. Planned comparisons showed that in each group, RTs were significantly slower when a distractor was present than when it was absent, both $p s<0.001$. Error rates were very low (high-fear group, $M=4.8 \%, S D=2.8$; low-fear group, $M=5 \%, S D=2.8$ ) and analyses did not show any significant effects.

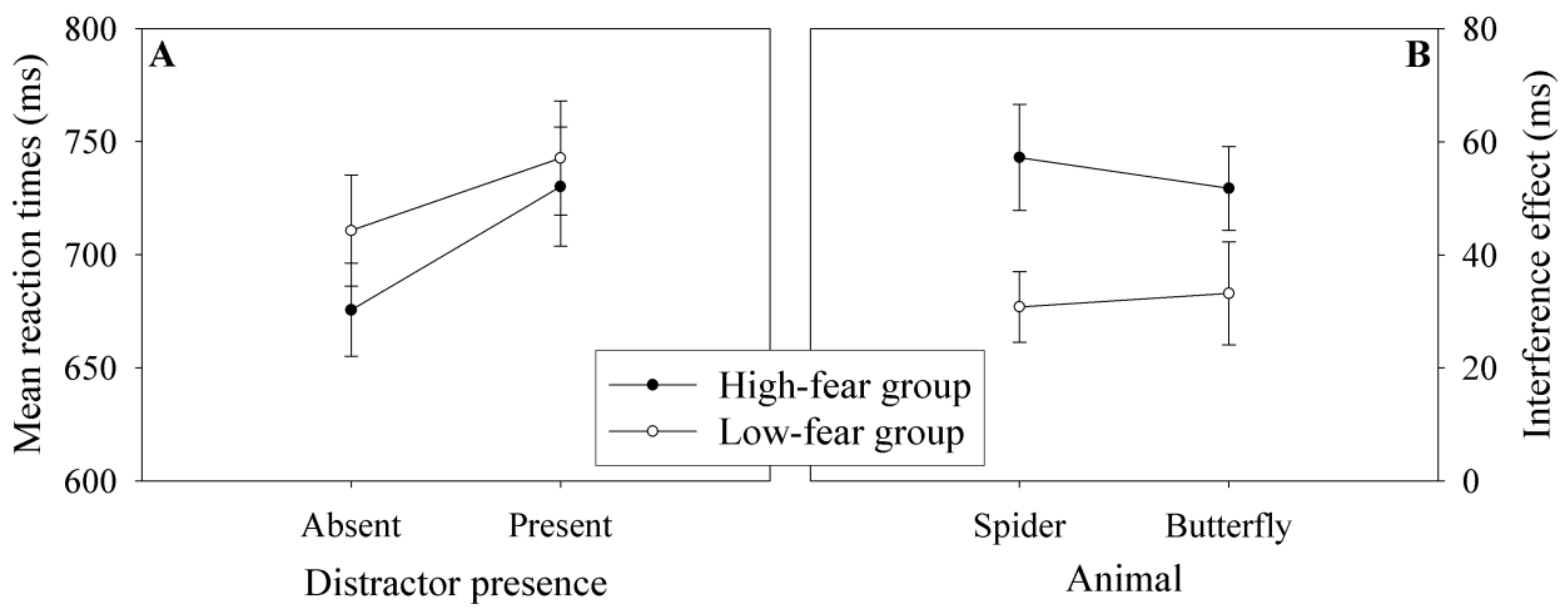

Figure 2. Results of Experiment 1: (A) Mean correct reaction times to the additional singleton task as a function of Distractor presence in high- and low-fear of spiders participants; (B) Mean interference effect as a function of the Animal presented in high- and low-fear participants. Error bars represent standard errors of the mean (SEM).

We then computed the interference effect produced by each animal by subtracting RTs to trials where no distractor was presented from trials with each type of distractor and conducted a two-way ANOVA with Group as between-subjects and Animal (spider, butterfly) as withinsubjects factors (see Figure 2B). There was a larger interference in high-fear $(M=54.5 \mathrm{~ms}$, $S D=40 \mathrm{~ms})$ than in low-fear participants $(M=32 \mathrm{~ms}, S D=37 \mathrm{~ms})$, as confirmed by a main 
effect of Group, $F(1,44)=5.42, p<0.05$. However, there was no main effect of Animal, $F<1$ and no significant interaction between Animal and Group, $F<1$.

\section{Perceptual discrimination task.}

Participants were able to quickly and accurately identify the content of both types of pictures (error rates less than 3\%). Overall, spiders were identified quicker than butterflies (531 versus $553 \mathrm{~ms}, F(1,46)=22.18 ; p<0.001)$, which could reflect a threat superiority effect (see Fox et al., 2007). However, this effect did not depend on whether participants had spider fear or not as there was no main effect of Group, $F<1$, and no interaction between Animal and Group, $F(1,46)=2.23 ; p=0.14$, which rules out the possibility that group differences in the additional singleton task result from an overall different ability to recognize insects between the two groups.

\section{Discussion}

The present results showed that people who fear spiders show larger attentional capture for both spiders and butterflies than people who do not fear spiders. This effect was unexpected since we anticipated that for high-fear participants only spiders would cause larger attentional capture. There are a few possible explanations for this effect. First, it is possible that high-fear participants have a general difficulty to inhibit all irrelevant information. Second, high-fear participants could have been captured by any salient visual event based on their expectancy of a spider since they knew that on any given trial, a spider or a butterfly were equally likely to appear as a distractor. Hence the mere expectation of the feared object could have led to an increase in capture by butterflies. Finally, the fear of spiders could have induced an extensive monitoring specific to insects and not other objects. These potential explanations were examined in Experiment 2.

\section{EXPERIMENT 2}

The goal of Experiment 2 was to clarify why in Experiment 1 participants with highfear of spiders were not captured more by the presence of their object of fear than by a neutral object. To control for the possible role of expectation of a spider we have consistently presented the same type of distractor throughout a block of trials. After a few trials of each block, participants should have realized that, whenever presented, a salient distractor always belonged to the same category and that it was either "safe" or "threatening". To check 
whether the monitoring extends only to insects, we included a third type of distractor that was not an animal (i.e. leaf).

We hypothesized that if high-fear participants have a general difficulty to inhibit irrelevant information, all three types of distractors would capture their attention more than for the low-fear participants. If, however, the fear of spiders induces an extensive monitoring of insects, then only spiders and butterflies, but not leaves should result in increased attentional capture. Finally, if it is the expectation of threat that boosts the attentional capture in high-fear people, only spiders should now interfere more with the search task, while the capture by the other objects should be equivalent to the low-fear group. In addition, to verify that our schematic stimuli did in fact elicit the expected emotions in the two groups, at the end of the session we asked participants to rate each of the distractors used in the experiment in terms of pleasance, arousal, fear and disgust.

\section{Method}

Participants.

We screened 240 new bachelor students in psychology and physiotherapy at the University of Liège. One hundred and twenty-two of them provided their details to be contacted for a later experiment. The selection procedure was the same as in Experiment 1. Twelve participants were included in the high-fear group (11 females, Mean age $=18.8$ years, $S D=1.2$; scores to the FSQ within the 75th percentile, i.e. 76) and 12 in the low-fear group (8 females, Mean age $=20.7$ years, $S D=2.6$; scores to the FSQ within the 30th percentile, i.e., 24).

\section{Stimuli and procedure.}

The procedure was the same as in Experiment 1 except that there was an extra category of neutral stimuli "leaves", which were symmetrical and had curved and spiky edges just as spiders and butterflies. We constructed 8 exemplars of leaves formed by the same average number of pixels as the animals and compared their salience to that of the animal stimuli during a pilot experiment conducted on 12 independent participants. We selected 4 leaves that were on average recognized with the same speed and accuracy as the animals. After a training consisting in 80 trials without distractor, each type of distractor appeared separately in a different block (whose order was counterbalanced across participants) composed of 40 trials 
with and 40 trials without a distractor. There were thus 240 experimental trials in total. Before each block participants were informed that the next block would be somehow different.

After the completion of the additional singleton task, participants rated each of the 12 stimuli on 5-points Likert scales as regard with pleasantness (1="very unpleasant"; 5="very pleasant"), arousal (1="not stimulating"; $5=$ ="extremely stimulating"), fear and disgust (1="no fear/disgust"; 5="extreme fear/disgust") they evoked. Finally, participants filled in the SBQ, the STAI-Y and the BDI.

\section{Results}

\section{Questionnaires.}

Results presented on Table 1B confirm that high-fear participants scored higher than low-fear participants on all aspects of spider fear. Table 1A indicates that high-fear participants were significantly more anxious than low-fear participants. The difference of state anxiety might be due to the experimental setting itself and a higher level of trait anxiety is not uncommon in spider phobia (see e.g. Olatunji, 2006). The levels of depression of the two groups only differed marginally.

\section{Stimuli ratings.}

Four ANOVAs with Group as between-subjects and Stimulus type (spider, butterfly, leaf) as within-subjects factors on mean ratings of pleasantness, arousal, fear and disgust all revealed significant interactions between Group and Stimulus type, $F(2,44)=5.1 ; p=0.01 ; F(2$, $44)=11.97 ; p<0.001 ; F(2,44)=23.71 ; p<0.001 ;$ and $F(2,44)=20.63 ; p<0.001$, respectively (see Table 2). Planned comparisons indicated that spiders were rated as more unpleasant, more arousing, more frightening and more disgusting by high-fear than by low-fear-participants (all ps <0.001). Moreover, spiders were judged more unpleasant, more arousing, more frightening and more disgusting than butterflies and leaves by high-fear participants (all $p \mathrm{~s}<0.001$ ). Lowfear participants judged spiders as more unpleasant, more disgusting (all $p \mathrm{~s}<0.001$ ) and more frightening (both $p \mathrm{~s}<0.002$ ) than butterflies and leaves and more arousing than leaves ( $p$ $<0.05)$. Therefore, schematic spiders elicited the expected emotional reactions since they were perceived as more negative than the neutral stimuli and more negative in the high-fear than in the low-fear group. Such schematic stimuli should thus be chosen over natural images since 
they obviously do not harm external validity while offering a much better control over lowlevel differences.

Table 2. Mean ratings of the three categories of stimuli in high- and low-fear participants of Experiment 2. Standard deviations are on italics.

\begin{tabular}{|c|c|c|c|c|c|c|c|}
\hline & & Spiders & & Butterflies & & Leaves & \\
\hline \multirow[t]{2}{*}{ Pleasance } & High-fear & 1.35 & 0.61 & 4.02 & 0.79 & 3.21 & 0.35 \\
\hline & Low-fear & 2.52 & 0.57 & 4.08 & 0.74 & 3.73 & 0.61 \\
\hline \multirow[t]{2}{*}{ Arousal } & High-fear & 4.35 & 0.9 & 2.13 & 0.84 & 1.83 & 0.84 \\
\hline & Low-fear & 2.73 & 1.08 & 2.27 & 1.21 & 1.94 & 0.92 \\
\hline \multirow[t]{2}{*}{ Fear } & High-fear & 4.25 & 1.1 & 1.35 & 0.46 & 1.17 & 0.31 \\
\hline & Low-fear & 2.02 & 0.88 & 1.02 & 0.07 & 1 & 0 \\
\hline \multirow[t]{2}{*}{ Disgust } & High-fear & 4.58 & 1.02 & 1.31 & 0.52 & 1.1 & 0.2 \\
\hline & Low-fear & 2.5 & 1.01 & 1.04 & 0.1 & 1.06 & 0.16 \\
\hline
\end{tabular}

Note. Pleasance was rated from 1 = "very unpleasant" to $5=$ "very pleasant" with 3 = "neutral". Arousal, fear and disgust were rated from $1=$ "no arousal/fear/disgust" to $5=$ "extreme arousal/fear/disgust".

\section{Additional singleton task.}

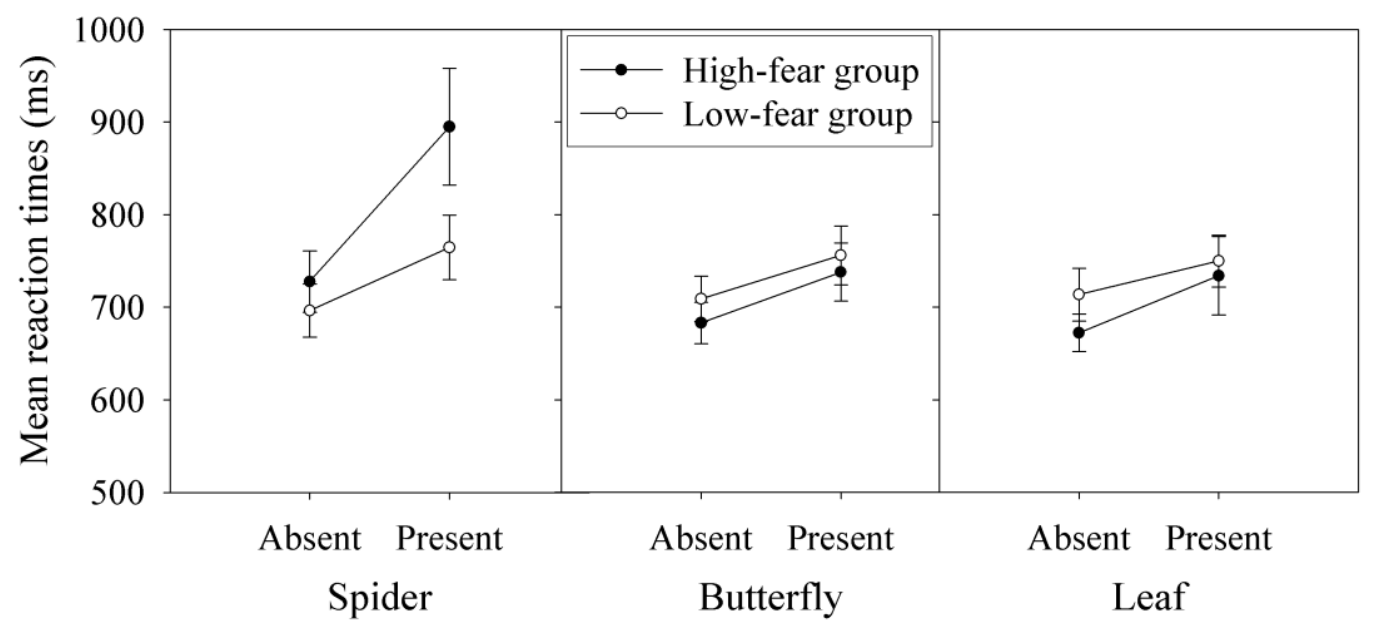

Figure 3. Results of Experiment 2: Mean correct reaction times to the additional singleton task as a function of Distractor type and Distractor presence in high- and low-fear of spiders participants. Error bars represent SEM.

A three-way mixed effects ANOVA with Group as between-subjects factor and Distractor presence and Distractor type (spider, butterfly, leaf) as within-subjects factors on mean correct RTs showed a main effect of Distractor presence, $F(1,22)=32.75 ; p<0.001$, and of Distractor type, $F(2,44)=4.6 ; p<0.05$ (see Figure 3 ). There was a significant interaction 
between Group and Distractor type, $F(2,44)=4.87 ; p<0.05$, resulting from high-fear participants being slower in the spider block than in the other two blocks (both $p s<0.01$ ). There was a Distractor type by Distractor presence interaction, $F(2,44)=9.29 ; p<0.001$; RTs in the no-distractor conditions in the three different blocks did not differ (all $p s>0.2$ ) but when a distractor was present, RTs were slower with spiders than with butterflies or leaves (both $p \mathrm{~s}<0.05)$. Finally, there was a three-way Group $\mathrm{x}$ Distractor type $\mathrm{x}$ Distractor presence interaction, $F(2,44)=3.53 ; p<0.05$. It should be noted that spider fear itself did not affect overall RTs, as there was no main effect of Group, $F<1$. To further analyze the three-way interaction, we computed the interference effect produced by the different distractors in each block by subtracting RTs on no-distractor trials from RTs on distractor trials (see Figure 4). We then used $t$-tests to compare the two groups on each distractor type. Spiders interfered more in high-fear than in low-fear participants, $t(22)=2.38 ; p<0.05$, whereas interference caused by butterflies and leaves did not differ between the two groups, both $t \mathrm{~s}<1$. Moreover, in high-fear participants, spiders interfered more than either butterflies or leaves, $t(11)=2.99$; $p<0.02$ and $t(11)=5.21 ; p<0.001$ respectively, which produced a similar level of interference, $t<1$. In low-fear participants, only spiders interfered more than leaves, $t(11)=2.52 ; p<0.05$, but there were no significant differences in interference between the other types of distractors, both $t \mathrm{~s}<1$.

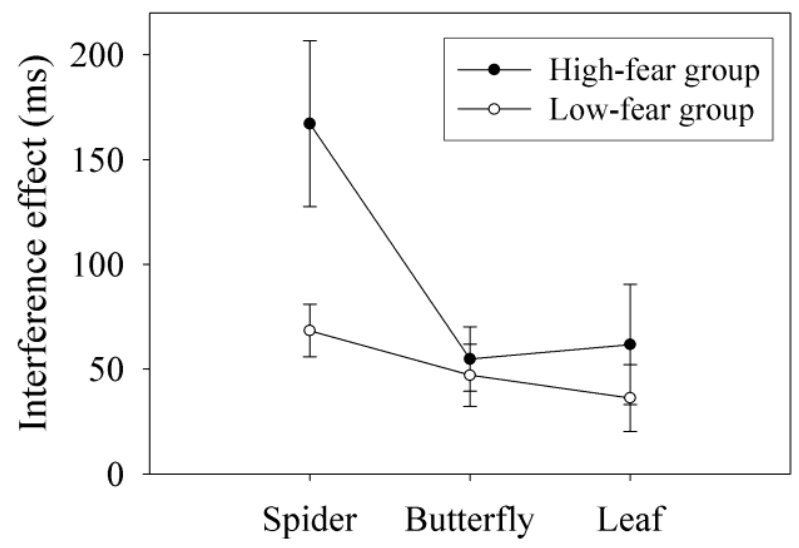

Distractor type

Figure 4. Results of Experiment 2: Mean interference effect as a function of Distractor type in high- and low-fear participants. Error bars represent SEM.

Error rates were overall very low (high-fear group, $M=3.9 \%, S D=2.9$; low-fear group, $M=$ $4.3 \%, S D=2.9$ ) and analyses did not show any significant effects. 


\section{Discussion}

This experiment shows that fear-related stimuli captured attention more than neutral stimuli, especially when people were scared of those stimuli and were sure when they can appear. In that situation, people who fear spiders showed a normal level of interference for the inanimate objects (i.e. leaves) and even with other animals (i.e. butterflies). People who do not fear spiders showed a similar interference with fear-related and with neutral animal stimuli and only showed a higher interference from spiders when they were compared to inanimate objects (i.e. leaves).

\section{GENERAL DISCUSSION}

The present study shows that people who fear particular stimuli (in this case spiders) monitor locations that potentially contain the objects that they fear in a compulsory fashion even though processing these objects is completely irrelevant for the current task (see also Notebaert, Crombez, Van Damme, De Houwer, \& Theeuwes, 2010).

The occurrence of this compulsory monitoring depends on the probability of a fearrelated object to appear. In Experiment 1, when a salient irrelevant distractor was equally likely to be a spider or butterfly, participants with a high spider fear were disrupted more by the presentation of both a spider and a butterfly than low fear participants. In Experiment 2, when the nature of a distractor was certain due to a blocked presentation people with a high fear of spiders could not help that their attention was captured by the occurrence of a spider (as evidenced by a very large capture effect of above $150 \mathrm{~ms}$ in the "spider" block). However, when the threat of the stimuli they fear was not present (i.e. in the "butterfly" and "leaf" blocks) no compulsory monitoring was observed anymore. As such one can conclude that among high fear participants capture occurs in a mandatory way whenever there is the possibility that something fearful is present.

Our study thus indicates that it is more the fear and the expectancy of a fear-related stimulus that drives attention rather than the visual features of the stimulus itself. In Experiment 1, the unpredictable appearance of spiders created an uncertain environment in which high-fear participants extensively monitored and got captured by all irrelevant salient visual events. This finding may be an empirical manifestation of what is known of clinically anxious individuals who display too much attention to aversive stimuli. Because they perceive 
the world as dangerous they constantly explore the environment for potential threats (Mogg \& Bradley, 1998; Williams et al., 1997) and have trouble inhibiting the processing of fearrelated information (see Weierich, Treat, \& Hollingworth, 2008). However, when high-fear participants knew the conditions under which a spider could be present (as in the blocked conditions of Experiment 2), their hypervigilance was limited only to the threatening conditions. In that case they could simply ignore objects that do not induce fear (butterflies or leaves) just as much as low-fear participants, which indicates that their attention is not captured by the features defining a spider themselves and that they do not undergo a general difficulty to inhibit irrelevant information but rather that they have a difficulty to deal with uncertain and threatening environments.

This tendency of high fear people to monitor the environment for threat might sometimes be beneficial, in cases where finding a feared object is required by the task. Indeed, previous studies showed that people that fear objects are quicker in finding them (see e.g., Lipp, 2006; Lipp \& Waters, 2007; Soares et al., 2009a, 2009b). The present study indicates that such effects might be mediated by the attentional and emotional set of the individual more than by the stimulus visual properties. The finding that expectancy of a feared object drives attentional selection also goes against the claim regarding the existence of a fear module independent of high-level processes (Mineka \& Öhman, 2002). It seems that mere expectation of a feared object and not just its presence in the scene increases the likelihood of it capturing attention (see Boston \& Sharpe, 2005; McGowan, Sharpe, Refshauge, \& Nicholas, 2009 for similar results with pain-related responses). Basically, it is the experience of fear and the expectation of the feared object that determines what objects receive priority in attentional selection.

The current findings are in line with the attentional control theory of Eysenck, Derakhshan, Santos and Calvo (2007) according to which anxiety has detrimental effect on attentional control settings. According to this theory anxious individuals preferentially allocate attention to threat-related stimuli which can be either internal (e.g. worries) or external (e.g. threat-related distractors). Crucially, the theory posits that anxiety impoverishes the goal-directed attentional system and increases the extent to which processing becomes dependent on the stimulus-driven attentional system. In the present study, the expectancy of a spider may have changed the direction of the goal-directed system of high fear participants and put the priority on stimulus-driven selection generating attentional capture by any 
information that may be threatening (Experiment 1). In Experiment 2 knowing that in a given block of trials the spider would not be present (but instead a butterfly or leaf) may have reduced the anxiety such that people now could rely more on the goal-directed attentional system. This reduction in anxiety allowed them to be goal-directed such that now they only selected the target instead of attention being captured by the more salient distractor.

Our results might also have important implications for the treatment of specific phobias and the understanding of the mechanisms involved in the existing treatments. For instance, the finding that unpredictable appearance of a frightening stimulus induces some attentional bias towards neutral stimuli could explain why phobic patients keep showing distress and hypervigilance even in the absence of the feared objects in uncertain situations. Moreover, the observation that high-fear participants are involuntarily disrupted from their current goal by the expectation of a spider might support the efficiency of attentional-training with phobic patients (i.e. train them not to search for frightening stimuli and perhaps not to attend them when they are actually present, see Weierich et al., 2008) to complement more classical exposure therapies. However, our participants were not formally assessed for spider phobia and these hypotheses remain to be tested on clinical populations.

To summarize, the current study shows that stimuli that are not relevant for your task may capture attention when there is a possibility that the stimulus that you fear is present in the environment. We explain these finding in terms of obligatory monitoring among people with high fear. Even though the feared stimulus is irrelevant the mere possibility that it could be present evokes automatic monitoring of locations that potentially may contain these fearful stimuli.

\section{Acknowledgments}

This research was supported by a grant from the F.R.S.-FNRS in which CD is a Postdoctoral Researcher. We thank Fanny Chapelier and Aline Cordonnier for their help during data collection. We thank Jim Enns and two anonymous reviewers for their comments on a previous version of this manuscript. 


\section{REFERENCES}

Arntz, A., Lavy, E., van den Berg, G., \& van Rijsoort, S. (1993). Negative beliefs of spider phobics: A psychometric evaluation of the spider phobia beliefs questionnaire. Advances in Behaviour Research and Therapy, 15, 257-277.

Beck, A.T., Rial, W.Y., \& Rickels, K. (1974). Short form of Depression Inventory: Crossvalidation. Psychological Reports, 34, 1184-1186.

Boston, A., \& Sharpe, L. (2005). The role of threat-expectancy in acute pain: effects on attentional bias, coping strategy effectiveness and response to pain. Pain, 119, 168-75.

Bruchon-Schweitzer, M., \& Paulhan (1993). Manuel de l'inventaire d'anxiété état-trait forme $Y(S T A I-Y)$. Paris: Éditions du Centre de Psychologie Appliquée.

Cave, K. R., \& Batty, M. J. (2006). From searching for features to searching for threat: Drawing the boundary between preattentive and attentive vision. Visual Cognition, 14, 629-646.

Delroisse, S., \& Philippot, P. (2007). Questionnaire sur la peur des araignées: validation française du "Fear of Spiders Questionnaire". Revue Francophone de Clinique Comportementale et Cognitive, 12(3), 14-21.

Eysenck, M.W., Derakshan, N., Santos, R., \& Calvo, M. (2007). Anxiety and cognitive performance: The Attentional Control Theory. Emotion, 7, 336 - 353.

Fox, E., Griggs, L., \& Mouchlianitis, E. (2007). The detection of fear-relevant stimuli: Are guns noticed as quickly as snakes? Emotion, 7, 691-696.

Gerdes, A., Alpers, G., \& Pauli, P. (2008). When spiders appear suddenly: Spider-phobic patients are distracted by task-irrelevant spiders. Behaviour Research and Therapy, 46, 174-187.

Gerdes, A., Uhl, G., \& Alpers, G. (2009). Spiders are special: fear and disgust evoked by pictures of arthropods. Evolution and Human Behavior, 30, 66-73.

Lang, P.J., \& McTeague, L.M. (2009). The anxiety disorder spectrum: Fear imagery, physiological reactivity, and differential diagnosis. Anxiety, Stress \& Coping, 22, 5-25. 
Lipp, O.V., \& Derakshan, N. (2005). Attentional bias to pictures of fear-relevant animals in a dot probe task. Emotion, 5, 365-369.

Lipp, O.V., Derakshan, N., Waters, A.M., \& Logies, S. (2004). Snakes and cats in the flower bed: fast detection is not specific to pictures of fear-relevant animals. Emotion, 4, 233 250.

Lipp, O.V. (2006). Of snakes and flowers: does preferential detection of pictures of fearrelevant animals in visual search reflect on fear-relevance? Emotion, 6, 296-308.

Lipp, O.V., \& Waters, A.M. (2007). When danger lurks in the background: Attentional capture by animal fear-relevant distractors is specific and selectively enhanced by animal fear. Emotion, 7, 192-200.

McGowan, N., Sharpe, L., Refshauge, K., \& Nicholas, M.K. (2009) The effect of attentional re-training and threat expectancy in response to acute pain. Pain, 142, 101-107.

Miltner, W.H.R., Krieschel, S., Hecht, H., Trippe, R., \& Weiss, T. (2004). Eye movements and behavioural responses to threatening and nonthreatening stimuli during visual search in phobic and nonphobic subjects. Emotion, 4, 323-339.

Mineka, S., \& Öhman, A. (2002). Phobias and preparedness: The selective, automatic, and encapsulated nature of fear. Biological Psychiatry, 52, 927-937.

Mogg, K., \& Bradley, B.P. (1998). A cognitive-motivational analysis of anxiety. Behaviour Research and Therapy, 36, 809-848.

Mogg, K., \& Bradley, B.P. (2006). Time course of attentional bias for fear- relevant pictures in spider-fearful individuals. Behaviour Research and Therapy, 44, 1241-1250.

Notebaert, L., Crombez, G., Van Damme, S., De Houwer, J., \& Theeuwes, J. (2010). Looking out for danger: An attentional bias towards spatially predictable threatening stimuli. Behaviour Research and Therapy, 48, 1150-1154.

Öhman, A., Flykt, A., \& Esteves, F. (2001). Emotion drives attention: detecting the snake in the grass. Journal of Experimental Psychology: General, 130, 466-478.

Olatunji, B.O. (2006). Evaluative learning and emotional responding to fearful and disgusting stimuli in spider phobia. Journal of Anxiety Disorders, 20, 858-876. 
Pflugshaupt, T., Mosimann, U.P., von Wartburg, R., Schmitt, W., Nyffeler, T., \& Müri, R.M. (2005). Hypervigilance-avoidance pattern in spider phobia. Journal of Anxiety Disorders, 19, 105-116.

Rinck, M., \& Becker, E.S. (2006). Spider fearful individuals attend to threat, then quickly avoid it: Evidence from eye movements. Journal of Abnormal Psychology, 115, 231238.

Rinck, M., Reinecke, A., Ellwart, T., Heuer, K., \& Becker, E.S. (2005). Speeded detection and increased discreased distraction in fear of spiders: Evidence from eye movements. Journal of Abnormal Psychology, 114, 235-248.

Soares, S., Esteves, F., \& Flykt, A. (2009a). Fear, but not fear-relevance, modulates reaction times in visual search with animal distractors. Journal of Anxiety Disorders, 23, 136144.

Soares, S., Esteves, F., Lundqvist, D., Öhman, A. (2009b). Some animal specific fears are more specific than others: Evidence from attention and emotion measures. Behaviour Research and Therapy, 47, 1032-1042.

Szymanski, J. \& O'Donohue, W. (1995). Fear of Spiders Questionnaire. Journal of Behavior Therapy and Experimental Psychiatry, 26, 31-34.

Theeuwes, J. (1991). Cross-dimensional perceptual selectivity. Perception \& Psychophysics, 50, 184-193.

Theeuwes, J. (1992). Perceptual selectivity for color and form. Perception \& Psychophysics, 51, 599-606.

Theeuwes, J. (2004). Top-down search strategies cannot override attentional capture. Psychonomic Bulletin \& Review, 11, 65-70.

Theeuwes, J. (2010). Top-down and bottom-up control of visual selection. Acta Psychologica, 123, 77-99.

Vrijsen, J.N., Fleurkens, P., Nieuwboer, W., \& Rinck, M. (2009). Attentional bias to moving spiders in spider fearful individuals. Journal of Anxiety Disorders, 22, 541-545. 
Waters, A.M., \& Lipp, O.V. (2008). The influence of animal fear on attentional capture by fear-relevant animal stimuli in children. Behaviour Research and Therapy, 46,114-121.

Weierich, M. R., Treat, T. A., \& Hollingworth, A. (2008). Theories and measurement of visual attentional processing in anxiety. Cognition \& Emotion, 22, 985-1018.

Williams, J. M. G., Watts, F. N., MacLeod, C., \& Mathews, A. (1997). Cognitive psychology and emotional disorders (2nd ed). Chichester: Wiley.

Yantis, S., \& Egeth, H. (1999). On the distinction between visual salience and stimulus-driven attentional capture. Journal of Experimental Psychology: Human Perception and Performance, 25 (3), 661-676.

Yantis, S., \& Jonides, J. (1984). Abrupt visual onsets and selective attention: Evidence from visual search. Journal of Experimental Psychology: Human Perception and Performance, 10, 601-621. 